Palabra Clave (La Plata)

ISSN: 1853-9912

palabraclave@fahce.unlp.edu.ar

Universidad Nacional de La Plata

Argentina

\title{
Ignacio Mancini. Lectura crítica de Ranganathan, S. R., Asundi, A. Y. and Meera, B. M. (2019). The science and the profession of libraries
}

Mancini, Ignacio

Ignacio Mancini. Lectura crítica de Ranganathan, S. R., Asundi, A. Y. and Meera, B. M. (2019). The science and the profession of libraries

Palabra Clave (La Plata), vol. 9, núm. 2, 2020

Universidad Nacional de La Plata, Argentina

Disponible en: http://www.redalyc.org/articulo.oa?id=350562513008

DOI: https://doi.org/10.24215/18539912e095

Esta obra está bajo una Licencia Creative Commons Atribución-NoComercial-Compartirlgual 4.0 Internacional. 
Ignacio Mancini. Lectura crítica de Ranganathan, S. R., Asundi, A. Y. and Meera, B. M. (2019). The science and the profession of libraries

Ignacio Mancini

Universidad de Buenos Aires. Facultad de Filosofia y Letras. Instituto de Investigaciones Bibliotecológicas, Argentina

ignaciomancini@gmail.com

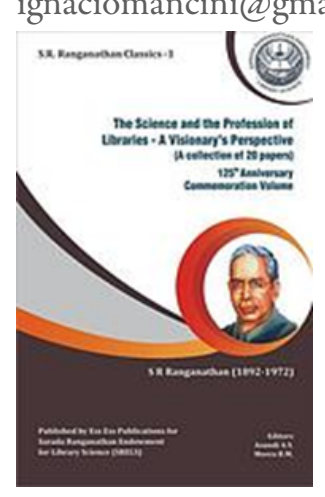

DOI: https://doi.org/10.24215/18539912e095

Redalyc: http://www.redalyc.org/articulo.oa? $\mathrm{id}=350562513008$

\begin{abstract}
Ranganathan S. R., Asundi A. Y., Meera B. M. The science and the profession of libraries: a visionary's perspective : a collection of 20 papers. 2019. New Delhi. Ess Ess Publications on Behalf of Sarada Ranganathan Endowment for Library Science. 324 p.pp.. 987-93-87698-09-3
\end{abstract}

Recepción: 11 Febrero 2020

Aprobación: 06 Marzo 2020

S. R. Ranganathan inmediatamente remite en el imaginario tanto a la Colon classification como a sus Five laws of library science. Tal vez sea menos conocido el hecho de que a lo largo de sus ochenta años de existencia, produjo más de 60 monografías y alrededor de 1.500 artículos (Bianchini, 2015). Resulta un auténtico desafío encontrar algún aspecto de la bibliotecología y cualquier elemento relacionado con la información documental que no haya sido abordada por Ranganathan. Para el centenario de su natalicio, tanto ISKO como IFLA centraron sus conferencias de ese año en su figura, y el gobierno de India lanzó una estampilla conmemorativa, marcando un hito en el reconocimiento social de la bibliotecología. Veinticinco años después, en octubre de 2017, varias entidades organizaron una conferencia internacional titulada "Revisiting Ranganathan", donde se percibe la necesidad de difundir su obra y aportes entre las nuevas generaciones de profesionales. Esta selección que aquí se reseña está integrada por veinte textos publicados en las siguientes revistas: Current science; Indian librarian; Special libraries; Libri; Annals of library science; Library herald; Herald of library science; y Library science with a slant to documentation, entre 1940 y 1968. ${ }^{1}$ La mayoría de estos títulos corresponden a publicaciones editadas en India. Se ha tomado como criterio que cada uno de los textos haya sido citado. La secuencia de aparición en la compilación responde a un criterio cronológico, pero dadas las características de su obra y a modo de propuesta, se alterará el orden elegido por los compiladores. ${ }^{2}$

Las cinco leyes de la bibliotecología sin duda resumen los fundamentos de la existencia y la práctica bibliotecaria. ${ }^{3}$ Han sido traducidas al japonés, hindú, portugués, italiano y coreano y aún en tiempos de internet, se destaca (Bianchini, 2015; Figueiredo, 1992; McMenemy, 2010; Pereira de Sousa y Targino, 2016; Takeuchi, 2010). La circulación y su fecha de postulación han tentado a otros autores a enarbolar otras nuevas leyes (Gorman, 1995; Line, 1996), que terminan constituyendo un truco publicitario por su estructura y falta de fundamentación. Surge entonces el interrogante: ¿cómo llega Ranganathan a formular las Five Laws?

En [Emergence of library service (Development of library science. 1) <1966> - pp. 213-240- LSwastD], Ranganathan nos lleva a la génesis del concepto desde lo irremediablemente vivencial. Emprende un viaje 
a Gran Bretaña en 1924. Allí tiene la oportunidad de visitar alrededor de 120 bibliotecas donde observa numerosas técnicas cuya aplicación desconocía. Llamativamente, muchos de los bibliotecarios británicos no tenían la oportunidad de conocer otras bibliotecas del Reino Unido, por lo que su plan le permitió obtener una visión global del estado del servicio bibliotecario en esas tierras. De vuelta en la India, Ranganathan prueba esas técnicas en la Universidad de Madras. Allí se organiza una conferencia en 1928 donde le ofrecen un espacio para exponer. La titula "Laws of library science". En esos meses de escritura, en una primera instancia, aparecen la segunda y la tercera ley como una unidad y, luego, emergen la cuarta y quinta ley. Intuye que la ley básica no estaba presente. Transcribe entonces un diálogo con el matemático Edward B. Ross. Ese intercambio atestigua que la primera ley de hecho no fue formulada por Ranganathan, sino por Ross.

Originalmente eran cuatro leyes, pero para ese momento en la Colon Classification, 4 era el dígito mnemónico para enfermedad. Es por ello que parte la segunda y la tercera y de esta manera se establece el 5, que es el mnemónico de Energía. Luego le agregaría como corolario de la cuarta ley "Ahorrar el tiempo del personal". En [Libraries and library movement <1940>-pp. 1-8 - Current science], marca su publicación como uno de los hitos del desarrollo de la bibliotecología en India, junto a las otras publicaciones de Madras Library Association (que funda él mismo), la revista Indian library journal, los primeros edificios de bibliotecas con estantería abierta y las primeras escuelas de bibliotecología. En ese mismo texto se pregunta si sería demasiado pretender que desde el gobierno y las universidades percibieran que el dinero gastado para alimentar los recursos de las bibliotecas y aumentar el personal, volvería a la comunidad bajo la forma de una alfabetización ampliada y una ciudadanía fortalecida. Con el tiempo, las ciencias comenzarían a percibir financiamiento en la recientemente independizada República de la India.

Ahora bien, ¿es posible formular una "ciencia" de las bibliotecas? Para adentrarse en la respuesta, hay que indagar en su texto [Library science and scientific method <1957> - pp. 49-76- AoLS] y preguntarse qué es una ciencia. Para Ranganathan la ciencia es la forma de nombrar a un dominio en el universo de conocimiento. Este desarrollo es posible gracias al método científico. Los múltiples dominios del universo de conocimiento se expresan a través de diversos métodos científicos. A continuación, Ranganathan desarrolla su compleja teoría del espiral del método científico, en el que los puntos cardinales son representados por los conceptos Nadir, Ascendente, Zenith y Descendente. La observación y experimentación que describen los hechos componen el Nadir. A través de la lógica inductiva y el cálculo matemático se llega a leyes empíricas o inductivas que componen el Ascendente. Por medio de la intuición y otros métodos se convierten en leyes fundamentales que representan el Zenith. La acumulación de estas leyes fundamentales conforman el Descendente. A su vez, la transición entre estos cuatro puntos cardinales está representada por un cuadrante. El ciclo de estos cuadrantes es el espiral del método científico. En cualquiera de estos cuadrantes puede haber una alteración, y allí comenzaría un nuevo espiral. En términos de Kuhn (1971), se podría decir que este espiral sería el motor dentro de un paradigma. Ranganathan aclara que habría una crisis en el método científico si persiste la contradicción entre los hechos empíricos de la experiencia y las leyes fundamentales.

Ciertamente, no sería arriesgado indicar que la recepción de este constructo teórico no ha sido extendida en la comunidad bibliotecológica internacional. Por un lado, Carlo Bianchini (2015) indica que para Ranganathan el hecho de haber incluido este texto en la segunda edición de las Five laws, lo considera parte de su obra cumbre. Por otro, el Sarada Ranganathan endowment for library science en 2006 reimprime la primera edición, entendiendo que la obra cumbre se completa en la primera. Trece años después, lo vuelven a publicar sin aclarar que forma parte de la segunda edición. En efecto, este escrito intenta actualizar algunas nociones. A continuación, se vincularán las actualizaciones de las Five laws con los otros textos de la compilación. Reafirma el valor de la primera ley: "Los libros están para usarse", cuya trivialidad y obviedad es necesaria para su formulación. Dentro de la descripción de individuos afectados por la desigualdad en el acceso a los libros, incluye considerar libros cenitales para personas postradas, libros para artesanos con ilustraciones para recientemente alfabetizados, audiolibros para iletrados, materiales audiovisuales para trabajadores fabriles que estén cumpliendo la jornada laboral y libros para especialistas que vivan lejos de los centros urbanos. 
El detalle sobre cómo se debería organizar un sistema bibliográfico nacional, delimitando responsabilidades desde el Estado hasta los lectores, es uno de los aspectos detallados en el desarrollo de la segunda ley en Five laws. Las propuestas de macro planificación se hacen presentes en el texto [Library development in India <1958> - pp. 115-124 - LH], donde propone tres sistemas de bibliotecas: el académico, el público y el productivo. ${ }^{4}$ Excluye explícitamente, por lo tanto reconoce, bibliotecas de archivos, manuscritos y para ciegos. En base a la población censada, calcula cuántas bibliotecas de cada tipo deberían estar en funcionamiento. ${ }^{5}$ Es este el texto donde toma posición en el debate sobre las cualificaciones del personal en bibliotecas escolares.

Sus ideas macro de un modelo de organización de bibliotecas públicas se materializa en la evaluación de un Proyecto de Ley [Model public libraries bill: an evaluation sponsored by the Union Ministry of Education <1964> - pp. 157-191 - LSwastD]. En este texto se puede observar a Ranganathan en su rol de evaluador. Se manifiesta en desacuerdo en numerosos puntos, ya sea por omisión, o supuestas imprecisiones del texto. Es de especial importancia que resalta que donde se piden libros "para el beneficio del pueblo", una maliciosa interpretación dejaría la puerta abierta para la censura. También se establece que no se debe prestar libros por fuera del edificio de la biblioteca. Puesto que era una evaluación formal, no se detuvo en el punto, pero es un claro ejemplo de la poca penetración de la First law. En cuanto a la selección, observa que debería dejar a las bibliotecas la posibilidad de elegir los materiales de acuerdo a sus necesidades. ${ }^{6}$

Ranganathan indica además que la especificación de que entre los servicios debe estar incluido el servicio de referencia es innecesaria. ${ }^{7}$ Pero la más grave de las faltas de este Proyecto de Ley sería la forma de financiamiento, puesto que al ser muy descentralizada permite el despilfarro de recursos.

La preocupación por la eficiencia en la ejecución de presupuestos está sumamente detallada en [Library grant to Indian universities: some suggestions for the $U G C<1957>-$ pp. 77-96-AoLS]. A los diez años de la independencia de la India, las universidades tienen que llegar a conquistar partidas destinadas a la investigación. Parte de este financiamiento está destinado a la compra de bibliografía. Pero al no sistematizar el proceso, se genera un cuello de botella en determinada época del año y no se puede cumplir a tiempo con la rendición. ${ }^{8}$ Ranganathan propone que un $20 \%$ de la partida sea destinada al personal requerido para elegir, procesar y poner a disposición el material. Estos bibliotecarios deben estar entrenados especialmente por profesores con dedicación exclusiva. La concepción del tiempo es semanal, puesto que las listas de novedades editoriales se publican una vez por semana. Cuando se centraliza en una época del año, se reduce o suspende el servicio a los lectores, puesto que la totalidad del personal se dedica frenéticamente a estas tareas. Incluso, en algunos casos no se llega a rendir las facturas por estas demoras y los bibliotecarios terminan arriesgando su trabajo por aprobar facturas ficticias para no perder el financiamiento. También sucede que compran únicamente lo que está disponible en librerías cercanas para simplificar el proceso. Cuando describe los requerimientos del personal para llevar a cabo esta tarea, en este texto se puede reconocer la excepcionalidad del pionero. A pesar de que él mismo admite haber sido de los primeros, se opone firmemente a que los profesores de las escuelas de bibliotecología sean de medio tiempo y que la otra mitad del tiempo trabajen en bibliotecas. Aquí es donde también expresa que la investigación en bibliotecología debe ser financiada, especialmente porque la India contribuyó al mundo con sus Five laws.

Otro de los textos en los que despliega su visión global es [Library responsabilities of independent India <1948> - pp. 15-23 - IL]. Un año después de la declaración de la independencia de la República de la India, Ranganathan destaca la oportunidad para que haya un sistema bibliográfico nacional despegado de ciertas tradiciones occidentales. Aquí se descubre una de las indicaciones para aplicar la Tercera ley: "A cada libro su lector", mas para el óptimo desarrollo de una nueva nación, desaconseja la difusión de lo que hoy conoceríamos como best-sellers y literatura de "moral subversiva".

Un país nuevo requiere de un plan para un sistema de bibliotecas, por lo que Ranganathan calcula la cantidad de bibliotecas necesarias agrupadas por tipología con pocos redondeos. Luego, fundamenta por qué 
debería haber 1000 bibliotecas industriales (bussiness libraries), 600 bibliotecas municipales departamentales, 15.000 bibliotecas escolares, 321 bibliotecas de distritos escolares, 1.000 bibliotecas de colleges, 30 bibliotecas universitarias y de investigación y una biblioteca nacional centralizada. Cabe destacar la idea de que el Bibliotecario Nacional cree bibliotecas con profesionales y materiales de la India en cada país, se estima que en embajadas o centros culturales.

Esta no sería la única aparición de la matemática en esta compilación. En la obra [Newton, mathematics and Ramanujan <1942> - pp. 9-14 - CS] presenta una comparación entre lsaac Newton y el matemático Srinivasa Ramanujan (1887-1920). No podía evitar relatar la anécdota del número 1729: cuando Ramanujan estaba en el hospital, recibió la visita de un colega llamado Godfrey Harold Hardy, ${ }^{9}$ que le comenta que venía en un taxi que tenía el número 1729 y lo hallaba un tanto soso. Ramanujan le comenta que para nada, puesto que es el número natural más pequeño que puede ser expresado como la suma de dos cubos positivos de dos formas diferentes $(1729=13+123=93+103)$.

Luego de describir el método científico, acuña el término bibliometría. En [Librametry and its scope <1967> - pp. 297-310 - LSwastD] relata la situación sucedida en 1948, en la que lo mencionan en un comentario a otro expositor, el Dr. Bernal, en el marco de una conferencia de ASLIB. Se presentan diversas instancias donde se aplicaría estadística, como calcular la carga total de trabajo por integrante del personal, con el objetivo de argumentar el aumento de la planta o las estadísticas horarias para saber cuántas personas se necesitan en cada sector a cada hora y, en el contexto de un sistema nacional, para saber cuántas sucursales es necesario abrir. También para ubicar los materiales en colecciones de acceso o depósitos según su uso, calcular el espacio necesario para las horas pico, mudanza de un edificio a otro sin afectar el servicio, ${ }^{10}$ y para calcular las inconsistencias posibles sin aplicar reglas de catalogación. ¿Funcionaría en la práctica? Para ello recomienda que se enseñe estadística en las escuelas de bibliotecología.

Al retomar la actualización de las Five laws en el desarrollo del método científico, con respecto a la Tercera ley, hace hincapié en el rol de la investigación para el desarrollo de las sociedades y la necesidad de que haya libros actualizados y accesibles para fortalecerla. No obstante, frecuentemente el concepto de libro resulta limitado, y tal como ya lo había mencionado en Five laws, la unidad de servicio a considerar debería ser artículos de revistas o incluso secciones o párrafos dentro de ellos. En este sentido, más allá de que no menciona a Paul Otlet en toda su obra, no reniega de la Documentación y afirma que el servicio de referencia especializado se denomina servicio de documentación. El micro-documento [Documentation: a vital link in communication (documentations in many lands 7) <1962> - pp. 149-156 - AoLS] es un ¿artículo? compuesto por un gráfico y una serie de cuadros. Es un intento didáctico de condensación de conceptos a la máxima expresión, pero dada su estructura seguramente sería rechazado en la mayoría de las revistas contemporáneas. En las ilustraciones se representa el ciclo documental y que su aplicación permitiría llegar a una mayor parte del conocimiento existente. Llamativamente, en el primer cuadro que trata sobre niveles de servicio, el nivel general lo ejecuta el bibliotecario referencista, mientras que el nivel medio (destinado a la gestión y la ingeniería productiva) lo lleva a cabo un information officer y un nivel más profundo dirigido al desarrollo y a la investigación aplicada, un documentalista.

Llaman la atención estas denominaciones, puesto que en [Revolutions in library science <1961> - pp. 139-147 - HoLS] afirma que quienes se escapan del término bibliotecarios bajo el mote de recuperadores de información o documentalistas, no comprenden las implicancias de una segunda revolución provocada por las Five laws y cuyo motor es el espiral del método científico. En consonancia con la historia oficial de la bibliotecología internacional, destaca a la fundación de ALA y a Melvil Dewey y Charles Ammi Cutter como los artífices de la primera revolución bibliotecaria llevada a cabo Estados Unidos a fines de siglo XIX. La obra de Ranganathan es sumamente autorreferencial, pero no por eso está exenta de contradicciones. Doce años antes, en [Special librarianship - What it connotes? <1949>, pp. 25-35] especifica que la bibliotecología especializada desde los sectores francófonos ha sido denominada documentación. Es preciso aclarar que Ranganathan era reacio a las lecturas en lenguas ajenas al inglés y las lenguas de la India (Sharma, 1979). Aquí 
brega por la penetración de la bibliotecología especializada en bibliotecas públicas. ${ }^{11}$ En $[$ Intellectualproblems and equipment consideration in the design of new systems <1959> - pp. 125-137 - LH], si bien observaba que no se podía delegar el análisis de los datos a las máquinas porque la cantidad de ocurrencias de una palabra en un texto no siempre denota la materia principal del mismo, ya se estaban desarrollando las primeras experiencias de extracción a través del lenguaje natural.

Ante este escenario, Ranganathan considera necesaria la función del especialista de datos. De hecho, este artículo trata ni más ni menos que de la minería de datos. Pero para llegar a ese concepto, profundiza sobre la naturaleza de la información textual que sentaría las bases de lo que sería el nuevo rol profesional: Ranganathan diferencia seis niveles de información textual. El primer nivel está representado por quienes sientan las bases de determinadas disciplinas o líneas teóricas, como Freud en el psicoanálisis o el mismo Ranganathan en la bibliotecología; el segundo a quienes dentro del sistema de investigación hagan un aporte realmente significativo como introducir terminología; el tercero a quienes dentro de la tarea de investigación aporten datos; el cuarto para la difusión de noticias; el quinto para las expresiones cotidianas; el sexto para un lenguaje adolescente que condensa lo coloquial en su máxima expresión, almacenado actualmente por los nuevos medios. ${ }^{12}$

Considera que los tres primeros son los que deberían organizarse y clasificarse de manera facetada. 13 En [Documentation, computer and classification <1967> - pp. 251-288 - LSwastD] detalla cómo las computadoras pueden trabajar con las facetas, luego de tipificar a los sistemas de clasificación y advertir sobre cierta inercia inmovilizadora que afecta tanto a bibliotecarios como a sus interlocutores del sistema productivo.

Con respecto al servicio de referencia, lo conceptualiza en [Reference service through four centuries $<1966>$ - pp. 241-249- LH]. A través de un recorrido histórico, recorre las posibilidades desde el autoabastecimiento en colecciones pequeñas y aisladas, hasta un servicio de referencia para resolver consultas puntuales, un servicio de información en bibliotecas públicas y un servicio de referencia de largo alcance en las universidades, con el apoyo de registros analíticos y bibliografías actualizadas. En el texto original de la First law desarrolla la necesidad de conocer la psicología del lector. Ahora los clasifica en función de si tienen complejo de superioridad, de inferioridad o traumático. Reafirma que en las bibliotecas especializadas se llama servicio de documentación, y que además requeriría generar contenidos corrientes y hacer pequeñas investigaciones temáticas. Luego presenta la experiencia del Documentation Research Training Center. En la transcripción de una serie de clases del DRTC [Evolution of reference service and documentation service <1965> - pp. 193-212 - LSwastD] se explicita la relación entre la documentación y el servicio de referencia. Ranganathan le confiesa a sus alumnos que él mismo quedó impactado con desagrado al toparse con el término por primera vez (sin embargo lo mantendría a lo largo de toda su obra al punto de escribir un libro sobre el servicio de referencia). Comenta como anécdota que la traducción literal al marathi quedaría como servicio de exigencia, por lo que propuso un término que se interpretaría como servicio que conduce a una integración armoniosa del lector y el libro. Reflexiona sobre la adopción de un abordaje constructivista de la enseñanza sin explicitarlo.

Cuando prosigue con la clase genera que los estudiantes lleguen a la conclusión de que servicio de referencia viene de obra de referencia y alude que su origen es la obra de James Wyers "Reference work", de 1930. Sin embargo, Samuel Swett Green (1876), ya hacía mención de un área de referencia. En el ejercicio, cuando les pregunta sobre otras formas de llamar al servicio de referencia, llega a servicio de información" y "servicio de consejos al lector. Ranganathan aclara que este último está en desuso porque es ofensivo al otorgarle un rol de superioridad al bibliotecario. Luego continúa en un ejercicio de periodización donde utiliza eventos significativos de la historia de la disciplina y de las coyunturas geopolíticas y lo restringe al análisis del Reino Unido, dado que posee una mayor tradición bibliotecológica. En el desarrollo del mismo se demuestra que al utilizar las fuentes, la educación tiene que estar basada en el uso de la biblioteca. 
Es en [Unified view of education and library work <1968> - pp. 311-316- LH] donde relata sus sinsabores con respecto a los métodos pedagógicos de sus primeros profesores para luego desarrollar un aprendizaje centrado en el estudiante y el uso de la biblioteca. Evidentemente desde la carencia de lo que podría ser y no es, Ranganathan desarrolla su teoría, ya que como estudiante no fue usuario de bibliotecas. El pragmático filósofo de la educación John Dewey fue su inspiración, de quien Ranganathan también fue su lector. La obra de ambos ha generado valiosos aportes en lo que respecta a la formación de usuarios en contextos educativos (Neelameghan, 1984).

Las actualizaciones de la Fourth law están centradas en su colorario: Ahorrar el tiempo del personal. En [Document, documentation and standardization <1957> - pp. 97-114 - AoLS], alienta la estandarización desde la producción misma de los documentos. Esta observación está en la línea de lo que luego serían los estándares de metadatos y digitalización. Ciertas sugerencias son difíciles de concebir en nuestra tradición latinoamericana, como la de los estándares para la construcción de índices analíticos.

Más allá de que se cuenta con la formación en la actualidad, la vasta producción editorial argentina sigue descuidando este valioso instrumento. Es notable observar que antes de la creación del hipertexto y las normas de accesibilidad, ya marcaba la necesidad de garantizar el derecho a la lectura para personas con discapacidad visual, a través de estándares. El contexto lingüístico que rodeaba a Ranganathan, un país con entonces doce lenguas oficiales, ilustra la necesidad de que haya normas de transliteración y títulos abreviados de revistas. Además, las actualizaciones de la Fifth law permiten incorporar el descarte de materiales al delimitar dos tipos de crecimiento. El ilimitado es el de las bibliotecas de conservación como las nacionales; mientars que las bibliotecas de servicio reemplazan a las "células muertas".

Las Five laws además justifican la investigación que se desarrolla en la disciplina. En [Is there a library profession? (musings on library service 2) <1968> - pp. 289-295 - LH] Establece varios puntos en común entre oficios y profesiones y también las diferencias, entre las cuales se encuentra la formación profesional de larga duración para el caso de las profesiones. Acude aquí al discutible esquema de la escala intelectual y afirma que los dos primeros cuartos en la escala son quienes se desempeñan en una profesión. Deberían poder juntar investigación empírica y a priori. Distingue los semiprofesionales de los profesionales, siendo los primeros quienes comparten el lugar pero no las tareas ni responsabilidades. La investigación que se desarrolla en la profesión se justificaría a partir de las cinco leyes. Se requieren habilidades intelectuales que luego redundarían en la escala salarial. Antes de diferenciar a profesiones de oficios, Ranganathan establece puntos en común y uno de ellos es el asociacionismo. [IFLA - what it should be and do <1954>, pp. 37-47, Libri] es un trabajo que surge a partir de un pedido de IFLA a Ranganathan tratando de detectar la necesidades de estos países que ahora están siendo ayudados por la UNESCO. Recibe con agrado esta amistosa invitación y a continuación realiza un ejercicio de honestidad brutal. IFLA es la Federación Internacional de Asociaciones de Bibliotecas. Ranganathan rápidamente nos sitúa en la acotada concepción de lo internacional, que se refiere principalmente a Norteamérica y Europa Occidental, excluyendo a Asia, Europa del Este, África, Sudamérica y Oceanía. ${ }^{15}$ Relata que cuando representantes de estos últimos continentes acuden a las reuniones de IFLA, quienes retienen el poder de IFLA eran usualmente tan corteses y condescendientes con ellos, como "benevolentes ricos hacia sus parientes pobres". Mientras desde la periferia no se trate de pelear por el poder, esto continuará. Es llamativo que Ranganathan publica sus Five laws en 1931 y recién desde 1948 acude a reuniones de IFLA. Reconoce que tal vez los participantes europeos y norteamericanos no sean concientes de su hegemonía, ni malintencionados, pero afirma que para los outsiders es tan claro como la luz del día en el trópico.

No obstante, la queja aislada resulta improductiva, por lo cual, a continuación (él como representante de los países no desarrollados), esboza ni más ni menos que una propuesta de organización de la IFLA y el primer paso es que en su órgano de gobierno haya representantes de estos continentes. De todos modos demuestra que la cólera no es su motor y admite que sería apresurado que los nuevos representantes soliciten bancas de acuerdo a la población de sus países, aunque su tasa de alfabetización podría contribuir a tomar esta decisión. 
Su racionalidad también le permite señalar que en estos casos, los sectores excluidos suelen formar otras instituciones y este no sería el camino a seguir. Naciones Unidas y sus agencias especializadas al menos tienen la cintura política que les permite ofrecerles la gestión de sectores no decisivos. Señala que Norteamérica y Europa no deberían tratar de imponer sus estándares. Por ejemplo, Japón había progresado mucho antes de la II Guerra Mundial, pero "se tuvo que tragar la píldora estadounidense". La juventud estaría libre del imperialismo y estas concepciones, así que la IFLA debería ser más receptiva a estas propuestas. ${ }^{16}$

Como es muy costoso organizarlas y acudir, la Conferencia General de IFLA debería realizarse una vez cada 5 años. Está bien que sea un espacio de socialización y que incluso tal vez haya intercambio de ideas útiles, si se le permite asistir a las generaciones jóvenes. Promueve las conferencias regionales. Con respecto a los viajes de los expertos de los países centrales, afirma que suelen hacer poco y utilizar una maquinaria publicitaria para justificar su viaje, y que ese sistema no beneficia a ninguna de las dos partes.

Cierra con un mensaje esperanzador, puesto que sería deseable concretar el intercambio intelectual y espiritual entre profesionales de todas partes del mundo y espera que IFLA se "cure" y que el beneficio de esta transformación lo pueda experimentar la próxima generación de bibliotecarios.

\section{REFERENCIAS}

Berndt, B. (1978). Ramanujan's notebooks. Mathematics magazine, 51(3), 147-164.

Bianchini, C. (2015). Ifondamenti della biblioteconomia: attuallità del pensiero di S. R. Ranganathan. Milano: Editrice Bibliografica.

Figueiredo, N. (1992). A modernidade das cinco leis de Ranganathan. Ciência da informação, 21(3). Recuperado de http://revista.ibict.br/ciinf/article/view/430

Gorman, M. (1995). Five new laws of librarianship. American libraries, 26(8), 784-785.

Green, S. S. (1876). Personal relations between librarians and readers. Library journal, 1(2-3), 74-81

Kuhn, T. (1971). La estructura de las revoluciones cientificas. Buenos Aires : Centro Editor de América Latina.

Line, M. (1996). Line's five laws of librarianship... and one all embracing law. The Library Association Record, 98(3), 144.

McMenemy, D. (2007). Ranganathan's relevance in the 21st century. Library Review, 56(2), 97-101.

Neelameghan, A. (1984). User education and user orientation: some issues with special reference to developing countries. En: New trends in international librarianship (pp. 234-238). New Delhi: Allied Pub.

Pereira de Sousa, M. y Targino, M. (2016). Cinco leis da biblioteconomia / Cinco leis de Ranganathan: resistindo bravamente ao tempo. Ciência da informação em revista, 3(1), 11-29. Recuperado de http://www.seer.ufal.br/i ndex.php/cir/article/view/2334

Ranganathan, S. R. (1961). Reference service. Bombay: Asia Publishing House.

Ranganathan, S. R. (1931). The five laws of library science. Madras: Madras Library Association. Recuperado de https ://catalog.hathitrust.org/Record/001661182

Ranganathan, S. R. (1963). The five laws of library science. Bombay: Asia Publishing House.

Satija, M. P. (1986). Five laws: the theory and philosophy of Library and Information Science. En: T. S. Rajagopalas (Ed.), Ranganathan's philosophy: assessment, impact and relevance (pp. 85-94). New Delhi: Vikas Publishing House.

Sharma, R. N. (1979). S. R. Ranganathan: a personal tribute. The Journal of library history, 14(1), 58-72.

Takeuchi, S. (2010). Dr. S.R. Ranganathan: a commentary on S. R. Ranganathan's five laws of library science. Information studies, 16(3), 149-154

Targino, M. G. y Sousa, M. E. P. (2016). As cinco leis de Ranganathan e gestão de bibliotecas universitárias. Revista FSA, 14(1), 57-58. DOI: http://dx.doi.org/10.12819/2017.14.1.3 
Venkatappaiah, V. y Kumar, P. S. G. (Comps.) (1994). Ranganathan dictionary: Indian terminology on library and information science. Delhi: B. R. Publishing Corporation

\section{Notas}

1 En el texto se abreviarán como, CS, IL, SL, Libri, AoLS, LH, HoLS y LSwastD. Los rangos de páginas corresponderán a la compilación.

2 Ranganathan ya en 1931 formula la Segunda ley de la bibliotecología como "Every reader his/her book". En vistas de que todavía no hay consensos sobre la forma de utilización del lenguaje inclusivo en la escritura académica, se utilizará el masculino para formas plurales con el fin de facilitar la lectura.

3 Las cinco leyes son: 1. Los libros están para ser usados, 2. A cada lector/a su libro, 3. A cada libro su lector, 4. Ahorrar el tiempo del lector, 5. La biblioteca es un organismo en crecimiento (Ranganathan, 1931, 1963; Satija, 1986).

4 Se ha optado por traducir como "bibliotecas productivas" a la expresión business libraries, puesto que además de las bibliotecas del sector comercial privado, incluye bibliotecas de esferas de gestión gubernamental.

5 A modo ilustrativo, estima la presencia de 40 bibliotecas universitarias y 300.000 centros de distribución en áreas rurales.

6 En Argentina, la CONABIP sigue esta línea.

7 La escasa cantidad de bibliotecas en Argentina con un área de referencia nos permite disentir.

8 CONICET es la institución análoga a UGC en Argentina. Sin embargo, en su relato Ranganathan no menciona como problema que los materiales comprados con fondos públicos no vuelvan al Estado

9 Hardy sería quien le diera los manuscritos de Ramanujan a Ranganathan para que los rescate en la Universidad de Madras. Han sido publicados luego de la muerte de Ranganathan. La historia de los cuadernos de Ramanujan está relatada en Berndt (1978).

10 Con este método habrían llevado a cabo una mudanza, cerrando el servicio solamente durante diez horas el día de la inauguración

11 En nuestros días en grandes bibliotecas públicas norteamericanas suscriben a bases de datos con colecciones de diarios o revistas populares. Invito a quien está leyendo estas líneas que trate de llegar a un artículo de revista popular en la biblioteca pública a la que acude como usuario.

12 Sesenta años después de que Ranganthan propusiera seis niveles de la información comunicacional y mencionara al data specialist se populariza el término big data. Sectores comerciales concentrados revolucionan la industria publicitaria e incluso se constituyen en actores claves en determinadas coyunturas electorales, gracias a la explotación de todos los niveles de la comunicación y la exaltación de los últimos tres en detrimento de los primeros

13 Resta preguntarse si ante la actual capacidad de procesamiento y almacenamiento de datos de las tecnologías digitales y las líneas de investigación de las ciencias de la comunicación estaríamos ante el mismo planteo.

14 Diecinueve años después se crea el ISSN y, en 1991, aparece la primera versión del Unicode

15 Cabe aclarar que América Central ni siquiera es mencionada por Ranganathan.

16 Paradójica y saludablemente, este texto está disponible en https://www.ifla.org/files/assets/hq/history/ranganathan_ 1954_libri.pdf

\section{BY-NC-SA}

